\title{
A novel approach regarding the anti-aging of facial skin through collagen reorganization
}

\author{
MARIA SIFAKI $^{*}$, DANIELA CALINA ${ }^{2 *}$, ANCA OANA DOCEA ${ }^{3 *}$, \\ SOTIRIS TSIOUMAS ${ }^{4}$, MARTHA-SPYRIDOULA KATSAROU ${ }^{5}$, SEVASTI PAPADOGIORGAKI ${ }^{6}$, \\ PERSEFONI FRAGKIADAKI ${ }^{7}$, DACIANA ELENA BRANISTEANU ${ }^{8}$, KONSTANTINOS KOUSKOUKIS $^{9}$, \\ JOHN TSIAOUSSIS $^{10}$ and DEMETRIOS A. SPANDIDOS ${ }^{11}$
}

${ }^{1}$ Private Practice Dermatologist-Venereologist, 71305 Heraklion, Greece; Departments of ${ }^{2}$ Clinical Pharmacy,

Dermatopharmacy and Cosmetology, and ${ }^{3}$ Toxicology, University of Medicine and Pharmacy of Craiova, 200349 Craiova, Romania; ${ }^{4}$ Private Practice Ophthalmologist, 11528 Athens; ${ }^{5}$ Research Group of Clinical Pharmacology and Pharmacogenomics, Faculty of Pharmacy, School of Health Sciences, National and Kapodistrian University of Athens, 15772 Athens; ${ }^{6}$ Teaching and Technical Support Staff, Laboratory of Electron Microscope, and ${ }^{7}$ Laboratory of Toxicology,

Medical School, University of Crete, 71003 Heraklion, Greece; ${ }^{8}$ Department of Dermatology, University of Medicine and Pharmacy 'Gr. T. Popa', 700115 Iasi, Romania; ${ }^{9}$ Department of Dermatology and Venereology, Medical School,

Democritus University of Thrace, 68100 Alexandroupoli; ${ }^{10}$ Laboratory of Anatomy-Histology-Embryology, and ${ }^{11}$ Laboratory of Clinical Virology, Medical School, University of Crete, 71003 Heraklion, Greece

Received October 18, 2019; Accepted November 26, 2019

DOI: $10.3892 /$ etm.2019.8254

\begin{abstract}
The needle shaping technique can be used to perform subcutaneous microtransplants, enabling the 'lifting' of the skin. This prospective cohort study aimed to examine the effects of needle shaping on facial skin tone, volume and histological structure. A total of 54 women underwent the needle shaping procedure performed by inserting a tiny acupuncture needle combined with mixed electrical currents. The overall treatment was completed within 4 sessions of 2 months apart, once every 15 days. Maintenance was ensured by 2 sessions (no longer than 15 days apart) every 6 months. Macroscopic skin appearance was evaluated by a specialized dermatologist and the satisfaction of the patients was assessed. The microscopic structure of the skin dermis was evaluated by optic and scanning electron microscopy. I-chrome staining
\end{abstract}

Correspondence to: Dr Daniela Calina, Department of Clinical Pharmacy, Dermatopharmacy and Cosmetology, University of Medicine and Pharmacy of Craiova, 2-4 Petru Rareș Street, 200349 Craiova, Romania

E-mail: calinadaniela@gmail.com

Dr Anca Oana Docea, Department of Toxicology, University of Medicine and Pharmacy of Craiova, 2-4 Petru Rareș Street, 200349 Craiova, Romania

E-mail: ancadocea@gmail.com

*Contributed equally

Key words: collagen reorganization, anti-aging effect, needle shaping, facial skin, new collagen synthesis demonstrated more compact dermis-collagen fibers which were larger and thicker as compared to the controls. Scanning electron microscopy demonstrated an increased dermis thickness as compared to pre-treatment. All patients that answered to the follow up reported satisfaction during assessment. The satisfaction of the patients was very good and excellent in $45 \%$ of cases. The results of the needle-shaping procedure are natural with no scaring or down time. Moreover, the result is lasting even for 1 year, depending always on the subject's lifestyle and general health condition.

\section{Introduction}

Skin is the largest body organ composed of the epidermis, dermis and hypodermis layer. Skin is heterogeneous, anisotropic and a non-linear viscoelastic material (1). Thus, the skin exhibits both viscous and elastic characteristics when undergoing deformation (2). The viscoelastic properties of the skin predominantly originate from its dermis compartment that is composed mostly of extracellular matrix sparsely populated by fibroblasts. Supporting cells in skin structures depends on a variety of factors, such as hyaluronan (HA), an unsulfated glycosaminoglycan that is an important component of the extracellular matrix network $(3,4)$ The dermis extracellular matrix consists mainly of collagen fibers (type I and type III), elastin and proteoglycans (1).

The mechanical properties of the skin largely depend on the nature and organization of dermal collagen and the elastic fibers network. Indeed, the collagens are responsible for the mechanical strength of the skin, whereas the elastic fibers bestow elasticity to the skin (5). The mechanical properties of the collagen matrix depend on the fine characteristics of the respective collagen types and their self-assembly as regards 
fiber length, fiber volume fraction and collagen molecular stretching, as well as the tilt angle of collagens determining its orientation (5). The diameters of papillary collagen fibers are in the range of 0.3 to $3 \mu \mathrm{m}(6)$, whereas those of reticular collagen are in the range of 10-50 $\mu \mathrm{m}$ (7). Importantly, the mechanical properties of the skin alter with aging, crucially contributing to the appearance of wrinkles and sagging skin. These changes are closely associated with the collagen matrix, as collagen fibers change with age, becoming denser and begin to 'unravel' (8).

There are several methods derived from classical acupuncture that are intended for the treatment of various skin or musculoskeletal conditions. The embedding method uses threads in subcutaneous tissue or muscles at specific points in order to treat musculoskeletal pain (9). Another variant uses micro-needles to create microwounds that stimulate fibroblast proliferation and dermal neovascularization, which is applied in the treatment of facial wrinkles (10). Needle-shaping uses very fine needles through which high tension and limited galvanic current are applied. This microsurgical technique, invented by Fippi in 2012 (11), has been established to be safe and able to physically reshape different body parts. Vibrance, the only medical device on the market able to perform needle shaping, has been found to improve the collagen matrix organization of aged skin. Specifically, the Vibrance machine uses 3 mixed currents which act synergistically, and thus result in a slight dehydration of the dermal elastic and collagen fibers, which in continuation, hook onto the needle and principally bind to each other. The respective currents are finely modulated at such an intensity so as to not be perceived by the patient, with the possible exception of particularly sensitive regions. To the best of our knowledge, no study to date has examined the effects of needle shaping on skin morphology and collagen structure. Thus, this was the aim of the present study.

\section{Patients and methods}

Patients. The group of patients recruited in this study consisted of 54 females in the age range (40-65 years) with varying extents of skin laxity. The study, including patient material, was performed in accordance with the Declaration of Helsinki (1964) in its latest amendment. Patient survey and clinical procedures were performed in accordance with the ethical approval 17/12.12.2016 provided by SkinClinic, Heraklion, Greece. All patients signed statements of informed consent for participating in the study.

Procedures. The patients underwent the needle shaping procedure in the period from January, 2017 up to January, 2018. Acupuncture needles sized $0.18 \times 40$ and $0.20 \times 60$ (a LKB 2088 Ultrotome $\mathrm{V}$ ) were used for the application of the needle shaping technique. The Vibrance machine uses 3 mixed currents imperceptible to patients. The electrical currents are programmable with basic parameters and their effect is enhanced by their synergic action. Four sessions of 4 treatments lasting 30 min were performed at day 0, 15, 30 and day 45. During the treatment, the women did not take hydrolyzed collagen orally and the skin care was basic, with moisturizing creams, and they did not receive creams or booster serum.
Macroscopic analysis and scoring. The macroscopic results of the procedure were evaluated by an independent dermatologist. Particularly, all patients were evaluated prior to the first session of treatment and 15 days after the final one (day 60). In addition, patients were asked to assess the outcome of the procedure in terms of personal satisfaction, by using the visual analog scale (VAS) at the same time. The assessment of satisfaction in this study was performed using the VAS score (12). A VAS for satisfaction is a horizontal line of $100 \mathrm{~mm}$ in length. At the beginning and at the end, there are 2 descriptors representing extremes of satisfaction (no satisfaction and extreme satisfaction). Patients rated their satisfaction by making a vertical mark on the $100-\mathrm{mm}$ line. The measurement in $\mathrm{mm}$ was converted to the same number of points ranging from 0 to 100 points. The exact question was 'Are you satisfied with your procedure?' A standard explanation of how to complete the VAS form was mentioned beneath the VAS horizontal line. Satisfaction on VAS was categorized as poor (range, 0-25), good (range, 26-50), very good (range, 50-75) and excellent (range, 76-100).

Histological analysis. A histological evaluation was performed on 5 female patients. Initially, a skin biopsy was obtained surgically from the skin behind the left ear (untreated area). At 15 days after the final session (day 60), a biopsy was taken in the treated skin area behind the right ear and examined by optical (pathologist) and scanning electron microscopy (SEM). For light microscopy (LM), small tissue blocks were fixed, both in $2 \%$ glutaraldehyde (Germiphene) and 2\% paraformaldehyde (Germiphene) in 0.1M cacodylate buffer, $\mathrm{pH} 7.4$ (Karnovsky's fixative, Electronic Microscopy Sciences), stained with $2 \%$ osmium tetroxide at room temperature for $2 \mathrm{~h}$ (Electronic Microscopy Sciences), dehydrated in a graded series of ethanol (Germiphene) and embedded in epoxy resin (Electronic Microscopy Sciences). Sections were cut on a LKB 2088 Ultrotome $\mathrm{V}$ microtome at a thickness of $1 \mu \mathrm{m}$, transferred to a water droplet on a gelatin-coated slide, and placed on a slide warmer plate at $50^{\circ} \mathrm{C}$. After drying, the sections were stained with a trichrome stain (Electronic Microscopy Sciences) according to a staining protocol for collagen fibres and viewed using a Nikon E800 light microscope. The samples were evaluated by a qualified pathologist who was unaware as to the identity of the samples.

For SEM, the Karnovsky-fixed tissue blocks were post-fixed with cacodylate buffered $2 \%$ osmium tetroxide ( $\mathrm{pH} 7.4$ ), for $2 \mathrm{~h}$, dehydrated in a graded series of ethanol, and dried using the critical point drying method with a BAL-TEC, CPD-O30 critical point dryer. The specimens were coated with gold, and viewed using a scanning electron microscope (JEOL JSM 7000F; JEOL Ltd.).

\section{Results}

Patient data. Data from 52 patients were available for analysis, since 2 patients missed follow-up. The results of this study demonstrated good to excellent construct validity. None of the patients had adverse effects with the minor exception of short-term redness and mild ecchymosis. Indeed, there was no downtime or side-effects associated with this treatment apart from some redness that lasted for half an hour. Ecchymosis in some cases may have lasted for a few days. 


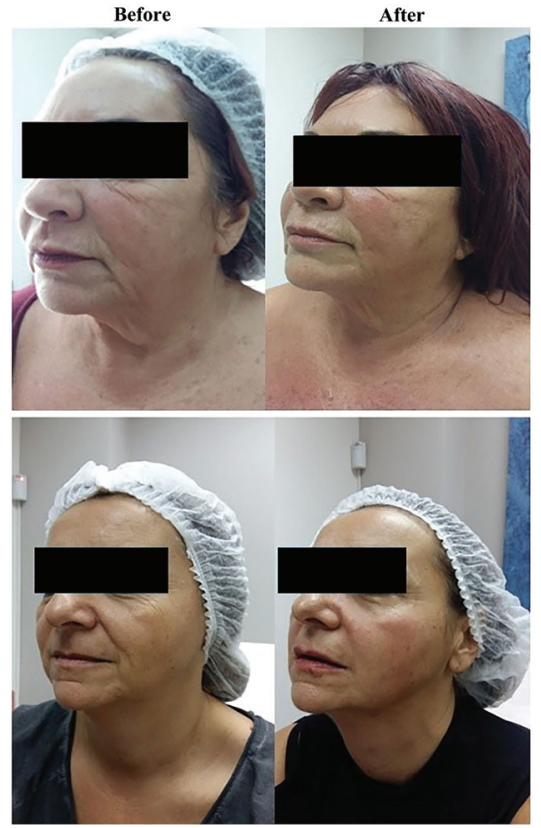

Figure 1. Clinical results of the needle shaping procedure before and after the treatment.
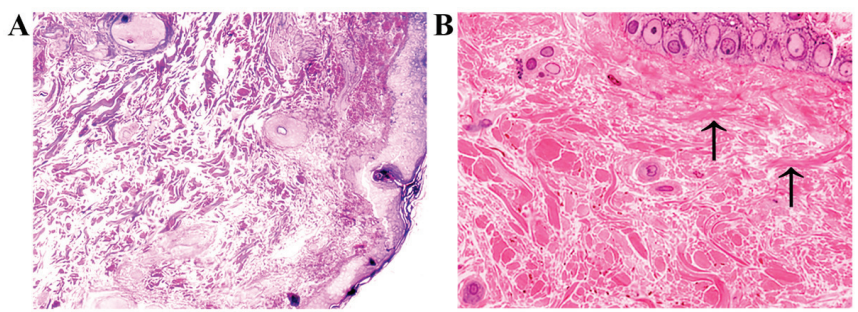

C

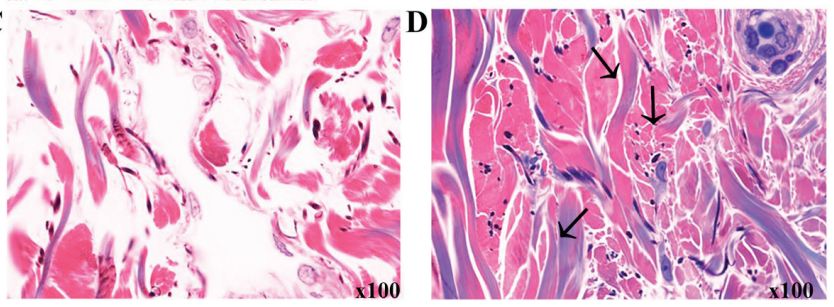

Figure 2. Histological evaluation by light microscopy of biopsies taken before and after needle shaping treatment. (A and C) Skin samples before (panel A, x25 magnification; panel C, x100 magnification) and (B and D) after needle shaping treatment (panel B, x25 magnification; panel D, x100 magnification) were stained with trichrome staining (most significant micrographs are shown). Changes in matrix organization are indicated by arrows. The images depicting tissues after the treatment (B and D) demonstrated a more compact dermis, with a healthy matrix network. Specifically, collagen fibers are larger and thicker with a more ordered alignment of fibers of the treated skin as compared to the images obtained before treatment (A and C).

Clinical assessment. Assessment by an independent dermatologist confirmed that skin laxity and tone had markedly improved in all patients resulting in a younger, fresher, healthier and brighter appearance of the patients' skin. Moreover, a reduction of wrinkles and fine lines was observed, as well as an increase in skin collagen and elasticity, with the skin looking more plumed and naturally 'lifted' without the aid of foreign substances that can sometimes alter facial features. As regards both criteria, in all cases, a significant improvement of skin tone was evident (Fig. 1).
Laboratory assessment. Moreover, in the case where a biopsy pre- and post-treatment was obtained, both the macroscopic, as well as histological characteristics of the skin were found to be significantly improved (Fig. 2).

Trichrome staining demonstrated a more compact dermis, with a healthy matrix network (Fig. 2B and D). Specifically, the collagen fibers were larger and thicker with a more ordered alignment of fibers of the treated skin as compared to the pre-treatment condition (Fig. 2A and C).

This finding was also confirmed by electron microscopy. The examination of the treated dermis by SEM demonstrated an increased dermis thickness (Fig. 3B) compared with the pre-treatment condition (Fig. 3A).

The examination of the dermis specimens by SEM at a higher resolution demonstrated that collagen the fibers before treatment were disorganized and of poorer quality (Fig. 4A and C). The needle shaping treatment enhanced collagen production, resulting in the formation of better organized, good quality fibers of a more ordered alignment (Fig. 4B and D).

Patient satisfaction. A total of 52 patients were available from the follow-up. The results demonstrated good to excellent construct validity. The results were as follows: Poor, 0 cases; good, 7 cases; (13.46\%); very good, 22 cases (42.30\%); and excellent, 23 cases (44.23\%) (Fig. 5).

\section{Discussion}

Rejuvenating the skin by stimulating the body's own regeneration mechanisms without surgery has an increasing popularity. Skin needling techniques are used in dermatology for various diseases: Post acne scars, particularly in severe forms resistant to conventional classical treatment $(13,14)$, re-pigmentation of hypopigmentation scars after burns, melasma, non-acne scars such us atrophic scars following varicella infection or to combat aging skin.

The results of this study demonstrated a good anti-aging effect and are comparable in efficacy with those of another study (15) that have used different micro-needling techniques. The results were analyzed 15 days after the end of the final session. The mode of action of needle shaping follows the mechanism of wound healing. The collagen production begins to recreate after the first 7 days of skin injury (15).

Fractional radiofrequency micro-needling has been used for skin rejuvenation in a group of 15 women. These were treated on one side of the face with monotherapy and in the control lateral part was added in addition stem cell conditioned medium. Fine wrinkles were reduced and the overall appearance was improved on the side where stem cells were added. From a histological point of view, an increased production of neo-collagen, similar to our study was observed with minimal side-effects: Mild erythema and procedural pain (16).

An ideal treatment for stimulating the production of collagen and elastin in the dermis is percutaneous collagen induction therapy. It uses a dermaroller, a cylindrical device fitted with needles, which is rolled back and forth and produces thousands of stings in the papillary dermis (17). Histological examinations demonstrated a considerable increase in collagen and elastin deposition, the epidermis has shown a thickening 


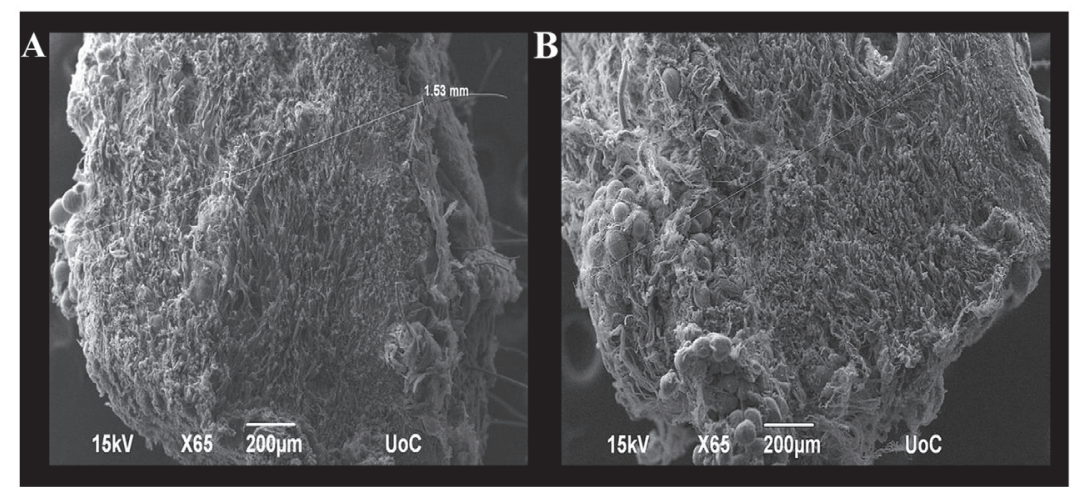

Figure 3. Evaluation by scanning electron microscopy (SEM) (most significant micrographs are shown). Skin specimens coated with gold (A) before and (B) after needle shaping treatment were viewed using a scanning electron microscope.

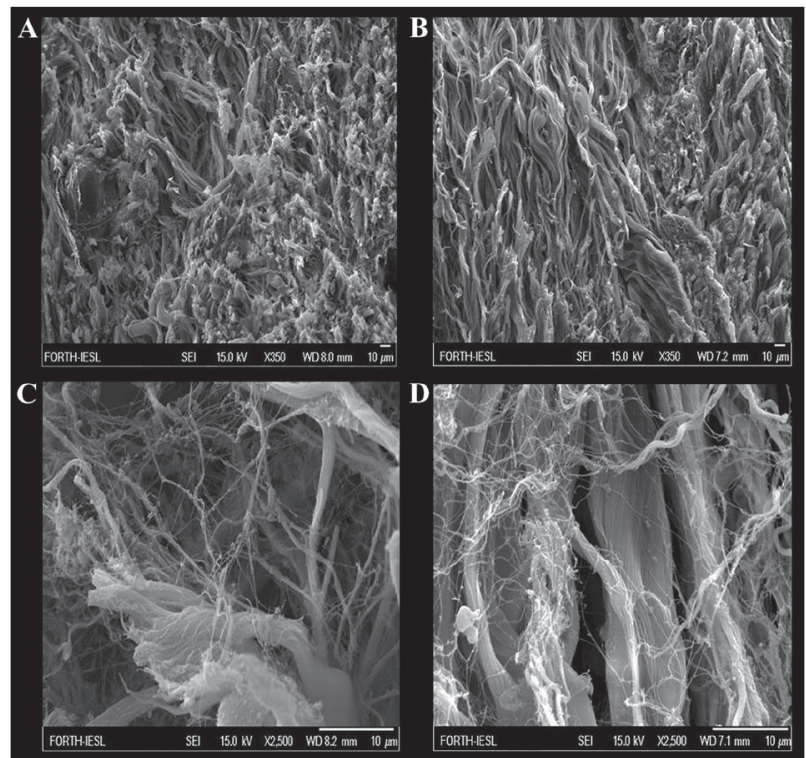

Figure 4. Evaluation by scanning electron microscopy (SEM). Skin specimens were coated with gold and viewed using a scanning electron microscope at different magnifications before (A and C) and after (B and D) needle shaping treatment were (most significant micrographs shown).

of the spinosum layer. However, the method involves more bleeding, is painful and requires local anesthesia (17).

Further studies are required in the future to confirm the current findings. These techniques may open new non-surgical horizons in aesthetic medicine, anti-wrinkle treatments and anti-aging treatments with improvements in skin quality.

The assessment of satisfaction is difficult as it is a multifaceted expression of affective, cognitive, physical, sensory, behavioral, social, cultural and subjective feelings. One of the potential methods of measuring satisfaction is by using the well-known VAS. The VAS satisfaction is a simple and valid instrument for the quantification of the satisfaction of the patient following aesthetic procedures; however, it cannot be used as the only outcome measurement. The VAS satisfaction is a useful addition to subjective and objective outcome measurement in documenting the results of the needle shaping.

In this study, all enrolled patients reported full satisfaction with the results. In general, the improvement of the treated

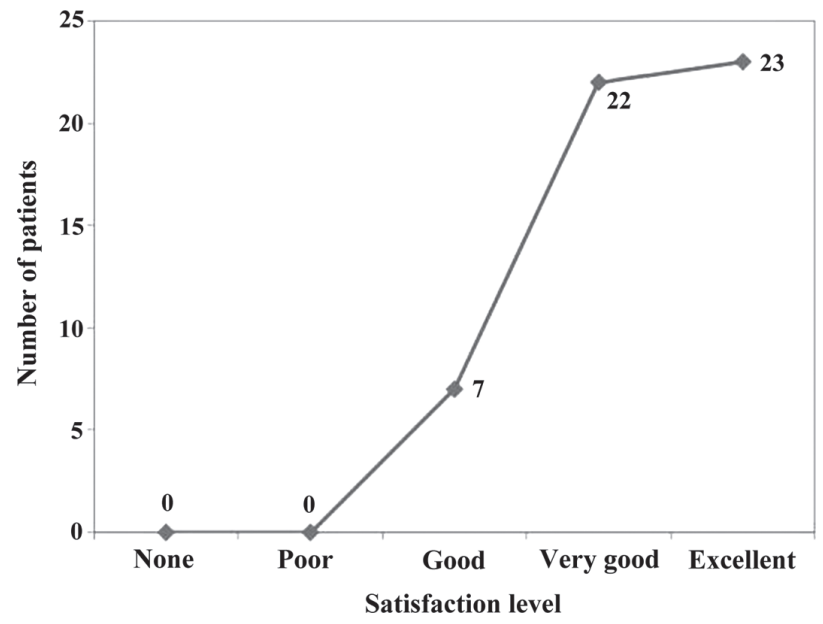

Figure 5. Distribution of the visual analog scale (VAS) satisfactionscore in the patient population.

skin area was dependent on the initial skin laxity and the requirements of the patient for perfect results.

However, satisfaction was not used only to measure the amount of improvement over time, but also to benefit from having knowledge of the patient's opinion regarding the clinical results. For this reason, we wished to have a more detailed documentation as to the following issues: How effective the treatment was and consequently how much the dermis area becomes affected after the needle shaping treatment has been performed.

In conclusion, needle shaping can be characterized as an auto-transplantation of tissue produced by our own body mimicking threads. Natural results are obtained and importantly, the patient physiognomy is not altered. Macroscopically, the results are visible from the very first treatment. The dermatological evaluation determined that skin laxity and tone had been improved, resulting in younger, fresher appearance of the facial skin. According to the findings of this study, the needle shaping technique has been proven to be effective and safe, followed only by very mild complications. The skin becomes, brighter, tighter of a visibly younger appearance, while microscopic examination revealed a denser dermis populated with larger, thicker and more correctly organized collagen fibers. Needle shaping is indicated as an innovative 
aesthetic strategy for improving skin tone and volume with zero side-effects.

\section{Acknowledgements}

Not applicable.

\section{Funding}

No funding was received.

\section{Availability of data and materials}

All data generated or analyzed during this study are included in this published article or are available from the corresponding author on reasonable request.

\section{Authors' contributions}

All the authors (MS, DC, AOD, ST, MSK, SP, PF, DEB, KK, JT and DAS) contributed equally to the conception and design of the study. MS, DC and DEB, KK contributed to sample collection. ST, SP, PF, JT and DAS worked and interpreted the histological evaluation and scanning electron microscopy. MA, DC, AOD and MSK drafted and wrote the manuscript. MA, DC, AOD, MK, DEB, KK and DAS provided advice on the experimental design, interpreted the results and critically revised the manuscript. All authors have read and approved the final version of the manuscript.

\section{Ethics approval and consent to participate}

Patient survey and clinical procedures were performed in accordance with the ethical approval 17/12.12.2016 provided by SkinClinic, Heraklion, Greece. All patients signed statements of informed consent for participating in the study.

\section{Patient consent for publication}

All patients agreed for their data to be published.

\section{Competing interests}

DAS is the Editor-in-Chief for the journal, but had no personal involvement in the reviewing process, or any influence in terms of adjudicating on the final decision, for this article. The other authors declare that they have no competing interests.

\section{References}

1. Ross MH, Kaye GI and Pawlina W: Connective Tissue in Histology: A text and Atlas. Lippincott Williams \& Wilkins, Pensylvania, PA, 2003.
2. Silver FH, Freeman JW and DeVore D: Viscoelastic properties of human skin and processed dermis. Skin Res Technol 7: 18-23, 2001.

3. Kavasi RM, Berdiaki A, Spyridaki I, et al: HA metabolism in skin homeostasis and inflammatory disease. Food Chem Toxicol 101: 128-138, 2017.

4. Nikitovic D, Berdiaki A, Galbiati V, Kavasi RM, Papale A, Tsatsakis A, Tzanakakis GN and Corsini E: Hyaluronan regulates chemical allergen-induced IL-18 production in human keratinocytes. Toxicol Lett 232: 89-97, 2015.

5. Agache PG, Monneur C, Leveque JL and De Rigal J: Mechanical properties and Young's modulus of human skin in vivo. Arch Dermatol Res 269: 221-232, 1980.

6. Brown RA, Shuttleworth CA and Weiss JB: Three new alpha-chains of collagen from a non-basement membrane source. Biochem Biophys Res Commun 80: 866-872, 1978.

7. Smith LT and Holbrook KA: Development of dermal connective tissue in human embryonic and fetal skin. Scan Electron Microsc (Pt 4): 1745-1751, 1982.

8. Lavker RM, Zheng PS and Dong G: Aged skin: A study by light, transmission electron, and scanning electron microscopy. J Invest Dermatol 88 (Suppl 3): 44s-51s, 1987.

9. Yun Y and Choi I: Effect of thread embedding acupuncture for facial wrinkles and laxity: A single-arm, prospective, open-label study. Integr Med Res 6: 418-426, 2017.

10. Cho JH, Lee HJ, Chung KJ, Park BC, Chang MS and Park SK: Effects of Jae-Seng Acupuncture Treatment on the Improvement of Nasolabial Folds and Eye Wrinkles. Evid Based Complement Alternat Med 2015: 273909, 2015.

11. Fippi G: Elettrochirurgia a flusso di elettroni convogliati. In: Trattato di medicina estetica. Massirone A (ed). Vol 1. 1st edition. Piccin-Nuova Libraria, Padova, 2010, pp1238-1249.

12. McCormack HM, Horne DJ and Sheather S: Clinical applications of visual analogue scales: A critical review. Psychol Med 18: 1007-1019, 1988 .

13. Ianoşi S, Ianoşi G, Neagoe D, Ionescu O, Zlatian O, Docea AO, Badiu C, Sifaki M, Tsoukalas D, Tsatsakis AM, et al: Age-dependent endocrine disorders involved in the pathogenesis of refractory acne in women. Mol Med Rep 14: 5501-5506, 2016.

14. Ianosi S, Neagoe D, Branisteanu DE, Popescu M, Calina D, Zlatian O, Docea AO, Marinas MC, Iordache AM, Mitrut P, et al: Comparative efficacy of oral contraceptive versus local treatment versus intense pulsed light combined with vacuum in endocrine acne in women. J Biol Regul Homeost Agents 32: 711-718, 2018.

15. Tsioumas S, Vlachodimitropoulos D and Goutas N: Clinical And Histological Presentation After Plexr Application, Needle Shaping (Vibrance) and O.F.F. Pinnacle Med Med Sci 2: 522-530, 2015.

16. Seo KY, Kim DH, Lee SE, Yoon MS and Lee HJ: Skin rejuvenation by microneedle fractional radiofrequency and a human stem cell conditioned medium in Asian skin: a randomized controlled investigator blinded split-face study. J Cosmet Laser Ther 15: 25-33, 2013.

17. Aust MC, Knobloch K, Reimers K, et al: Percutaneous collagen induction therapy: an alternative treatment for burn scars. Burns 36: 836-843, 2010.

This work is licensed under a Creative Commons Attribution-NonCommercial-NoDerivatives 4.0 International (CC BY-NC-ND 4.0) License. 